\title{
Adesão à higiene de mãos entre profissionais de um serviço de pronto atendimento
}

\author{
Adhesion of hand hygiene by professionals in the emergency care of a university hospital
}

\author{
Adriana Cristina de Oliveira ${ }^{1}$, Adriana Oliveira de Paula ${ }^{2}$, \\ Mariana Almeida de Souza ${ }^{3}$, Alanna Gomes da Silva ${ }^{4}$
}

Oliveira AC, Paula AO, de Souza MA, Silva AG. Adesão à higiene de mãos entre profissionais de um serviço de pronto atendimento / Adhesion of hand hygiene by professionals in the emergency care of a university hospital. Rev Med (São Paulo). 2016 out.-dez.;95(4):162-7.

RESUMO: Objetivo: avaliar a taxa de adesão à higiene de mãos de uma equipe multiprofissional. Materiais e métodos: estudo transversal, realizado em uma unidade de pronto atendimento de um hospital universitário de Belo Horizonte-MG, entre maio e agosto de 2013, por meio de observação direta das equipes médicas e de enfermagem. As observações se basearam nos cinco momentos para higienização das mãos da Organização Mundial de Saúde, utilizando-se um instrumento estruturado. Os dados foram analisados no SPSS, realizando-se análise descritiva e univariada. Resultados: registraram-se 931 oportunidades de higienização das mãos, $88,9 \%$ entre os técnicos de enfermagem, $6,9 \%$ médicos, $3,7 \%$ enfermeiros e $0,5 \%$ para auxiliares de enfermagem, obtendo-se uma taxa de adesão global de $19,4 \%$. A higienização simples com água e sabão foi mais utilizada pelos profissionais $(47 \%)$. Conclusão: a taxa de adesão à higienização das mãos foi considerada baixa, observando-se a necessidade e treinamentos para melhoria da mesma.

Descritores: Infecção hospitalar; Higiene das mãos; Segurança do paciente; Serviços médicos de emergência.
ABSTRACT: Objective: to evaluate hand hygiene compliance among healthcare workers. Methodology: cross-sectional study, conducted in an emergency department of a university hospital in Belo Horizonte, between May and August 2013, which evaluated through direct observation, the rate of adherence to hand hygiene by healthcare workers. The observations were based on the five moments for hand hygiene indicated by the World Health Organization. data were analysed using spss software, by descreptive and univariated analysis. Results: 931 opportunities for hand hygiene were registered, $88.9 \%$ among nursing technicians, $6.9 \%$ among doctors $3.7 \%$ for nurses and $0.5 \%$, nursing assistants, giving a globaL rate of adherence among professionals of $19.4 \%$. Handwashing with soap and water was the most frequent metod used by professionals (47\%). Conclusion: the rate of adherence to hand hygiene was considered low, which is worrying ahead to the relevance of such action in infection control.

Keywords: Cross infection; Hand hygiene; Patient safety; Emergency medical services.

1. Enfermeira. Pós-Doutora pela Universidade de Nova York. Professora Associada do Departamento de Enfermagem Básica da Escola de Enfermagem da Universidade Federal de Minas Gerais. Belo Horizonte (MG), Brasil. Orientador. E-mail: acoliveira@gmail.com.

2. Enfermeira. Doutora pela Universidade Federal de Minas Gerais Belo Horizonte (MG), Brasil. E-mail: adrianaopaula@gmail.com

3. Graduada em enfermagem pela Universidade Federal de Minas Gerais Belo Horizonte (MG), Brasil. E-mail: mari.almeidas@yahoo. com.br

4. Mestranda em enfermagem pela Universidade Federal de Minas Gerais Belo Horizonte (MG), Brasil. E-mail: alannagomessilva@ gmail.com

Endereço para correspondência: Adriana Oliveira de Paula. Avenida Alfredo Balena, 190. Santa Efigênia, Belo Horizonte, MG. CEP: 30130-100 


\section{INTRODUÇÃO}

Organização Mundial de Saúde (OMS) desde 2002 tem voltado sua atenção para melhorar a segurança dos cuidados de saúde prestado aos pacientes. Neste sentido, tem buscado mobilizar esforços em todo o mundo, através dos "Desafios Globais" que constitui uma das estratégias que visa prevenir os eventos adversos de saúde ${ }^{1}$.

Dentre os eventos adversos na saúde, destacam-se as infecções relacionadas à assistência à saúde (IRAS), por ser um dos grandes problemas enfrentados pela saúde pública, não só no Brasil, mas também em nível mundial. Tais infecções são aquelas adquiridas pelo individuo durante o período em que o mesmo esteja submetido à assistência profissional. Pode ocorrer em âmbito domiciliar, ambulatorial, hospitalar, dentre outros ${ }^{2-3}$.

Dentre as medidas de controle e prevenção das IRAS, destaca-se a higienização das mãos (HM), por ser uma medida simples, porém, de grande impacto e importância, sendo comprovadamente eficaz na prevenção e redução das infecções, uma vez que impede ou reduz a transmissão de infecções de um paciente para outro ${ }^{1,4-6}$.

Diante disto, a OMS lançou o primeiro desafio mundial pela segurança do paciente, que teve como tema "Uma Assistência Limpa é Uma Assistência Mais Segura", e objetivo a conscientização e compromisso político dos países membros da OMS sobre a importância da higiene das mãos para prevenir infecções associadas aos cuidados de saúde ${ }^{7}$.

Apesar da conhecida importância da HM, estudos apontam que nem sempre ela é realizada pelos profissionais na frequência e qualidade apropriada, o que se torna um grande desafio para a segurança do paciente e redução de eventos adversos, como as IRAS ${ }^{4,8}$.

Neste contexto, torna-se necessário monitorar a adesão a HM dos profissionais, para avaliar a necessidade de estratégias de melhoria fundamentadas em treinamentos e, sobretudo, para propor indicadores de feedback desta prática às equipes assistenciais para o conhecimento de sua performance.

Com base no exposto, o objetivo desse estudo foi de verificar a adesão à higienização de mãos entre a equipe multiprofissional em uma unidade de pronto-atendimento.

\section{MATERIAIS E MÉTODOS}

Tratou-se de um estudo transversal, que avaliou a taxa de adesão à higienização de mãos pelos profissionais de uma unidade de pronto atendimento de um hospital universitário de grande porte, localizado em Belo Horizonte, Minas Gerais, entre maio e agosto de 2013.

O pronto atendimento desse hospital conta com quatro enfermarias, cada uma delas, com seis leitos, além de um quarto destinado a paciente em isolamento de contato, com dois leitos.
Foram elegíveis como população de estudo os profissionais que prestavam assistência direta ao paciente, médicos ou profissionais de enfermagem (técnicos e enfermeiros), e que concordassem participar do estudo após apresentação e assinatura do Termo de Consentimento Livre Esclarecido (TCLE). Foram excluídos aqueles profissionais que se encontravam de folga, férias ou licença durante o período de coleta de dados.

A coleta dos dados foi realizada por estudantes de graduação e de doutorado previamente treinados. $\mathrm{O}$ treinamento fundamentou-se na leitura dos seguintes materiais: Manual para Observadores: Estratégia Multimodal da OMS para a melhoria da higienização de mãos; Guia para Implantação: Um Guia para a implantação da estratégia multimodal da OMS para a melhoria da higienização das mãos e Segurança do paciente. Higienização de Mãos, seguido de discussões e esclarecimentos de dúvidas. Os alunos foram liberados para coleta após atingir um nível de concordância de no mínimo $85 \%$ com o pesquisador principal.

Após esta etapa, a equipe da pesquisa iniciou o trabalho em campo, propriamente dito, para observações fundamentadas nas cinco indicações para realização da HM de acordo com a OMS: "Antes de contato com o paciente", "Antes de realizar procedimentos assépticos", "Após risco de exposição a fluidos corporais", "Após contato com o paciente" e "Após contato com as áreas próximas ao paciente" . Cada profissional foi observado até atingir um número de no mínimo vinte oportunidades, por duas vezes em dias distintos.

A observação foi realizada utilizando-se um instrumento que continha as seguintes variáveis: nome do observador, data, dia da semana, setor, turno, categoria profissional, sexo e código do profissional observado (iniciais dos nomes para preservar a identidade do participante). Também constava no instrumento os cinco momentos indicados para a realização da HM, de acordo com a OMS e o tipo de HM realizado (água e sabão e/ou fricção antisséptica).

Com o objetivo de tentar minimizar o efeito Hawtorne, principal desvantagem das observações diretas, os profissionais de saúde, através do TCLE foram informados que seriam observados no que diz respeito à higienização das mãos, porém não tinham conhecimento de quem iria realizar a observação e quando. No momento da coleta de dados, os observadores não informavam o real motivo de sua presença, sendo identificados apenas como alunos de graduação.

Os dados foram tabulados e analisados no programa estatístico Statistical Package for Social Science (SPSS). Realizou-se então uma análise descritiva, apresentandose médias e desvio-padrão para variáveis quantitativas, além de porcentagens e valores absolutos para variáveis qualitativas. Utilizou-se o teste qui-quadrado para análise univariada. Considerou-se associação estatística um valor de $\mathrm{p}<0,05$. 
O projeto respeitou os aspectos éticos da pesquisa em humanos de acordo com a Resolução de 466 de 2012, sendo aprovado pelo comitê de ética em pesquisa da Universidade Federal de Minas Gerais, sob parecer número (CAAE: 18477913.1.0000.5149).

\section{RESULTADOS}

Foram observados 44 funcionários, não havendo nenhuma recusa em participar do estudo. Obteve-se pela observação direta um total de 926 oportunidades, sendo que a maior parte ocorreu durante a semana $(75,3 \%)$ e final de semana $(24,7 \%)$.

O turno observado com maior frequência foi o da manhã $(75,6 \%)$, seguido pela tarde $(21,9 \%)$ e pela noite $(2,5 \%)$. As observações dos turnos da manhã e tarde tinham uma duração média de quatro horas $(08 \mathrm{~h}$ às $12 \mathrm{~h}$ ou $14 \mathrm{~h}$ às $18 \mathrm{~h}$, respectivamente), sendo que as observações do turno noturno duravam em média três horas (19 as $22 \mathrm{~h}$ ).

Em relação à demanda de atividades realizadas junto aos pacientes, os técnicos de enfermagem foram os mais observados, representando um total de 828 oportunidades $(89,4 \%)$, seguidos pelos médicos (64 oportunidades $6,9 \%$ ) e enfermeiros (34 oportunidades - 3,7\%).

No que se refere à taxa de adesão à HM, encontrouse uma taxa global de adesão de 19,2\%, sendo que a Tabela 1 resume as variáveis relacionadas à adesão dos profissionais.

Tabela 1. Taxa de adesão dos profissionais de acordo com o dia da semana, turno, categoria profissional e momento da assistência. Belo Horizonte, MG, Brasil, 2013

\begin{tabular}{lccc}
\hline \multirow{2}{*}{ VARIÁVEIS } & \multicolumn{2}{c}{ ADESÃO (\%) } & VALOR \\
\cline { 2 - 2 } & SIM & NÃO & DE P \\
\hline Dia da semana & 16,9 & 83,1 & 0,003 \\
$\begin{array}{l}\text { Dias úteis } \\
\text { Final de semana }\end{array}$ & 26,2 & 73,8 & \\
Turno da observação & & & \\
Diurno & 18,5 & 81,5 & $<0,001$ \\
Noturno & 47,8 & 52,2 & \\
Categoria profissional & & & \\
$\begin{array}{l}\text { Enfermeiro } \\
\text { Médico }\end{array}$ & 44,1 & 55,9 & 0,000 \\
$\begin{array}{l}\text { Técnico } \\
\text { Momento assistencial }\end{array}$ & 20,3 & 79,7 & \\
$\begin{array}{l}\text { Antes de tocar o } \\
\text { paciente }\end{array}$ & 18,1 & 81,9 & \\
$\begin{array}{l}\text { Antes de procedimento } \\
\text { asséptico }\end{array}$ & 23,0 & 77,0 & \\
$\begin{array}{l}\text { Após risco de contato } \\
\text { com fluidos }\end{array}$ & 33,0 & 67,0 & \\
$\begin{array}{l}\text { Após contato com o } \\
\text { paciente }\end{array}$ & 10,0 & 80,0 & \\
$\begin{array}{l}\text { Após contato com as } \\
\text { superfícies }\end{array}$ & 12,1 & 87,9 & \\
\hline
\end{tabular}

De acordo com os dados mostrados pela Tabela 1, observou-se que para todas as variáveis foi encontrada diferença estatística, indicando que os profissionais nos finais de semana, no turno da noite, enfermeiros e após risco de contato com fluidos realizaram HM com maior frequência.

Em relação ao momento assistencial para realização da HM, observou-se uma diferença entre as categorias profissionais, apresentada na Tabela 2.

Tabela 2. Distribuição do momento assistencial de acordo com a categoria profissional. Belo Horizonte, MG, Brasil, 2013

\begin{tabular}{lcccc}
\hline \multirow{2}{*}{$\begin{array}{l}\text { Momento da } \\
\text { HM }\end{array}$} & \multicolumn{2}{c}{ Categoria profissional (\%) } & \multicolumn{1}{c}{$\begin{array}{l}\text { Valor } \\
\text { de p }\end{array}$} \\
\cline { 2 - 4 } & $\begin{array}{l}\text { Enfermeiro } \\
(\mathrm{n}=34)\end{array}$ & $\begin{array}{l}\text { Médico } \\
(\mathrm{n}=64)\end{array}$ & $\begin{array}{c}\text { Técnico } \\
(\mathrm{n}=828)\end{array}$ & \\
\hline $\begin{array}{l}\text { Antes de tocar } \\
\text { o paciente }\end{array}$ & 38,2 & 51,6 & 30,6 & 0,000 \\
$\begin{array}{l}\text { Antes de } \\
\text { procedimento }\end{array}$ & 8,8 & $-*$ & 14,9 & \\
asséptico \\
$\begin{array}{l}\text { Após risco de } \\
\text { contato com } \\
\text { fluidos }\end{array}$ & 8,8 & $-*$ & 11,4 & \\
$\begin{array}{l}\text { Após contato } \\
\text { com o paciente }\end{array}$ & 35,3 & 42,2 & 29,1 \\
$\begin{array}{l}\text { Após contato } \\
\text { com as } \\
\text { superfícies }\end{array}$ & 8,8 & 6,3 & 14,1 \\
\hline
\end{tabular}

* Durante os momentos de observação, nenhum médico realizou procedimento asséptico ou teve risco de contato com fluidos.

Diante dos dados apresentados na Tabela 2, observa-se a diferença do cuidado assistencial entre os profissionais e distintas categorias no setor estudado. A quase totalidade das oportunidades observadas entre os médicos estiveram relacionadas a contatos antes e após paciente, sem realização de procedimentos assépticos ou risco de contato com fluidos. Os profissionais que mais realizaram procedimentos assépticos e tiveram risco de contato com fluidos foram os técnicos de enfermagem.

No que diz respeito aos tipos de HM, higienização simples com água e sabão ou fricção antisséptica com solução alcoólica, foi avaliada a frequência com que os profissionais aderiram a cada tipo mediante observação das oportunidades analisadas (Figura 1).

Observou-se que os profissionais do PA realizaram a HM com água e sabão com maior frequência, independente do momento assistencial e da categoria profissional. 


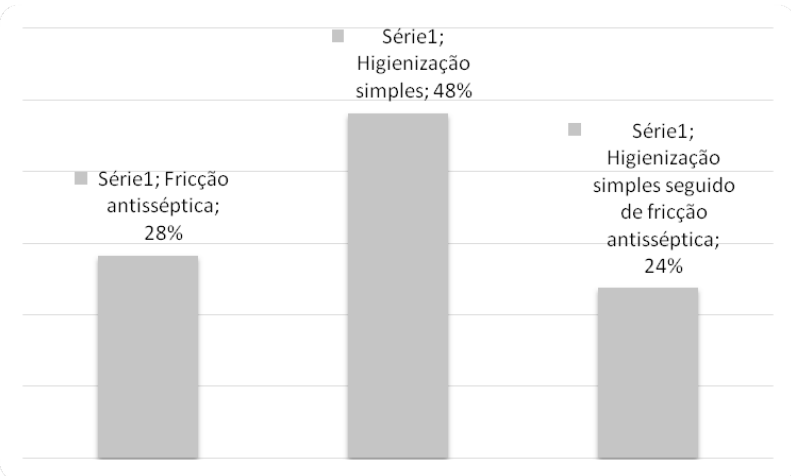

Figura 1. Porcentagem de realização de cada tipo de higienização de mãos. Belo Horizonte, MG, Brasil, 2013

\section{DISCUSSÃO}

Apesar da higienização das mãos, no contexto do cuidado assistencial ser uma medida conhecida frente ao controle de infecções, observou-se neste estudo uma baixa adesão dos profissionais de saúde a esta prática de forma semelhante à reportada por outros estudos, não ultrapassando $50 \% 0^{8-9}$.

Os pronto-atendimentos, caracterizados pelos serviços de emergência, constituem a porta de entrada das instituições hospitalares de forma geral. Nesta modalidade de atendimento, a Portaria 2.048 de 2002 do Ministério da Saúde do Brasil recomenda que os pacientes permaneçam neste local por no máximo 24 horas, considerando o objetivo de resolutividade deste setor. Porém, o encaminhamento dos casos às unidades assistenciais é quase sempre dificultado, sobretudo, pela falta de vagas em UTI para os pacientes graves, levando à manutenção do paciente por período superior ao recomendado no PA. Esta realidade ocasiona uma superlotação e, com isso, maior dificuldade de que uma assistência adequada e livre de riscos seja oferecida/disponibilizada aos pacientes. Tal situação não é encontrada em outros estudos internacionais, em que os pacientes permanecem por curtos períodos de tempo das salas de emergências, sendo transferidos para outras unidades com maior rapidez e fluxo garantido ${ }^{10-11}$.

A superlotação, associada a outras características, tais como, clientela diversificada e alta demanda de leitos são frequentemente associadas à colonização de pacientes, risco de contaminação cruzada, e, consequentemente, aumento da morbidade e mortalidade dos mesmos ${ }^{12-13}$. Assim enfatiza a importância de trabalhos voltados para o controle de IRAS nestes setores.

Em relação à categoria profissional, os enfermeiros obtiveram a maior taxa de adesão neste estudo. Um estudo realizado pelo Hospital das Clínicas de Porto Alegre mostrou uma adesão à HM por enfermeiros de quase $80 \%$, o que é de suma importância, uma vez que estes profissionais ocupam posição de líderes de equipe, o que os tornam exemplos a serem seguidos ${ }^{14}$.

Por outro lado, chama atenção o achado de que os médicos obtiveram taxas maiores que os técnicos de enfermagem, porém menores que os enfermeiros. No entanto, estes resultados podem divergir de outros estudos que apontam a equipe médica com menores taxas de adesão à HM, quando comparados a outros profissionais de saúde ${ }^{15-16}$.

Contudo, ressalta-se que tais comparações devem levar em consideração as metodologias adotadas nos diferentes estudos, unidades analisadas, especialidade da equipe médica, dentre outros fatores que podem influenciar nas taxas de adesão, tais como a menor frequência de contato desses com os pacientes, tipos de contato e procedimentos realizados, etc.

Os técnicos e auxiliares de enfermagem foram os profissionais com as menores taxas de adesão. Vale destacar que estes passam a maior parte do tempo com os pacientes, quando comparados aos demais profissionais da equipe de saúde, além de realizarem diversos cuidados assistenciais. Assim, são frequentemente relacionados a um risco aumentado de disseminação de microrganismos pelo elevado contato com o paciente, considerando a importância das mãos na disseminação cruzada de microrganismos.

$\mathrm{O}$ momento assistencial em que o profissional está envolvido pode ser diretamente relacionado com a prática ou não da higienização de mãos. No que diz respeito ao cuidado direto ao paciente, a adesão a HM ocorreu em maior proporção após risco de contato com fluidos corpóreos, o que pode ser justificado pelo profissional perceber, com mais clareza, o risco de contrair uma infecção cruzada e a maior necessidade de higienizar as mãos. Em contrapartida, antes do contato com o paciente, apresentou uma taxa de adesão menor.

Esse dado vem de encontro a outros estudos que indicam que a prática de higienização de mãos, geralmente, ocorre em maior frequência quando relacionada à proteção pessoal e, em menor taxa como prática de prevenção para o paciente pr-18. $^{17}$.

O momento após tocar superfícies obteve a menor taxa de adesão à HM deste estudo. Este fato pode estar relacionado à falta de conhecimento dos profissionais de que a superfície também pode servir como uma fonte ou reservatório do microrganismo, tendo um importante papel na transmissão de infecções ${ }^{1}$. Além disso, muitas vezes ocorrem os contatos desnecessários com as superfícies, em que os profissionais tocam biombos, leitos, portas e outros equipamentos e áreas da unidade do paciente, sem ao menos perceber esse contato, assim, não tendo para si estas situações como oportunidades para realizar a HM. 
Oliveira AC, et al. Adesão à higiene de mãos entre profissionais de um serviço de pronto atendimento.

Quando executada a HM, há controvérsias na literatura quanto ao tipo mais utilizado pelos profissionais, variando de acordo com a localização, tipo de estudo e intervenções. Em alguns estudos o uso da higienização simples foi mais frequente, atingindo taxas superiores a $90 \%$, sendo que em outros, a fricção antisséptica com solução alcoólica teve um maior uso, sendo influenciadas por intervenções para melhoria da $\mathrm{HM}^{17,19,21}$.

O uso de sabonete líquido é recomendado quando as mãos estão visivelmente sujas, porém, quando não há sujidade, a fricção alcoólica deve ser utilizada, frente à sua eficácia, baixa exigência em infraestrutura, pouco tempo para aplicação e boa tolerância da pele. De tal forma, é interessante o incentivo da prática de higienização por fricção alcoólica, respeitando as indicações específicas para cada tipo de higienização ${ }^{3}$.

Destaca-se, no Brasil, a obrigatoriedade da disponibilização de preparação alcoólica, em local visível e acessível para que a fricção antisséptica das mãos: seja realizada nos pontos de assistência de todos os serviços de saúde do Brasil, independente de sua complexidade ${ }^{22}$.

\section{CONCLUSÃO}

Percebeu-se que a taxa de adesão à higienização das mãos por profissionais assistenciais de uma unidade de pronto-atendimento de um hospital universitário apresentou-se menor em dias úteis, nos horários matutino e vespertino, entre os técnicos de enfermagem e após contato com superfícies. Destaca- se ainda que os profissionais realizaram a HM simples com mais frequência do que a fricção antisséptica.

Tais resultados reforçam a ideia de que é imprescindível que sejam realizadas ações voltadas para a conscientização dos profissionais e, principalmente, mudança de prática dos mesmos, tais como estratégias multimodais variadas e periodicamente, como intervenções, palestras, fixação de cartazes, entre outros, para o aumento da adesão a higiene de mãos.

Participação dos autores na concepção do artigo: Adriana C. Oliveira: Autora orientadora responsável pelo planejamento, correção e análise crítica dos dados e da redação e aprovação da versão final; Adriana $O$ de Paula: Autora principal responsável pela concepção, redação e revisão, além da obtenção e análise crítica dos dados; Mariana A. de Souza: Autora responsável pela concepção, redação e revisão; Alanna G. da Silva: Autora responsável pela redação e revisão.

\section{REFERÊNCIAS}

1. World Health Organization (WHO). WHO guidelines on hand hygiene in health care- First Global Patient Safety Challenge Clean Care is Safer Care. Geneva, 2009. Available from: http:// apps.who.int/iris/bitstream/10665/44102/1/9789241597906_ eng.pdf.

2. Cristina AO, Oliveira AP. Infecções relacionadas ao cuidar em saúde no contexto da segurança do paciente: passado, presente e futuro. REME. 2013 jan/mar; 17(1):216-20. doi: $10.5935 / 1415-2762.20130018$.

3. Brasil. Agência Nacional de Vigilância Sanitária. Segurança do paciente: higienização das mãos. Brasília; 2009. Disponível em: http://www.anvisa.gov.br/servicosaude/ manuais/paciente_hig_maos.pdf.

4. Marra AR, Moura DF, Paes AT, Santos OFP, Edmond MB. Measuring rates of hand hygiene adherence in the Intensive Care Setting: A comparative study of direct observation, product usage, and electronic counting devices. Infect Control Hosp Epidemiol. 2010;31(8):796-801. doi: 10.1086/653999.

5. Brasil. Agência Nacional de Vigilância Sanitária. Higienização das mãos em serviços de saúde. Brasília; 2007. Disponível em: http://www.anvisa.gov.br/hotsite/higienizacao_maos/ manual_integra.pdf.

6. Oliveira AC, Oliveira AP. Monitoração da adesão à higienização das mãos: uma revisão de literatura. Acta Paul Enferm. 2011;24(3):407-13. http://dx.doi.org/10.1590/S010321002011000300016
7. Sax H, Allegranzi B, Chraïti MN, Boyce J, Larson E, Pittet D. The World Health Organization hand hygiene observation method. Am J Infect Control. 2009;37(10):827-34. doi: 10.1016/j.ajic.2009.07.003.

8. Alsubaie S, Maither AB, Alalmaei W, Al-Shammari AD, Tashkandi M, Somily AM, et al. Determinants of hand hygiene noncompliance in intensive care units. Am J Infect Control. 2013;41(2):131-5. doi: 10.1016/j.ajic.2012.02.035.

9. Guedes M, Miranda FMA, Maziero ECS, Cauduro FLF, Cruz EDA. Adesão dos profissionais de enfermagem á higienização das mãos: uma analise segundo o modelo de crenças em saúde. Cogitare Enferm. 2012;17(2):304-9. doi: 10.5380/ce.v17i2.27886.

10. Porter AL, Wyrick D, Bowman SM, Recicar J, Maxson RT. The effectiveness of a statewide trauma call center in reducing time to definitive care for severely injured patients. J Trauma Acute Care Surg. 2014;76(4):907-11. doi: 10.1097/ TA.0000000000000142.

11. Edwards R, Hutson R, Johnson J, Sherwin R, GordonStrachan G4, Frankson M, et al. Severe sepsis in the emergency department - an observational cohort study from the university hospital of the West Indies. West Indian Med J. 2013;62(3):224-9. Available from:http://caribbean.scielo. org/scielo.php?pid=S004331442013000300012\&script $=$ s ci_arttext\&tlng=en.

12. Oliveira AC, Clemente WT, Lucas TC, Martinho GH. 
Oliveira AC, et al. Adesão à higiene de mãos entre profissionais de um serviço de pronto atendimento.

Nosocomial infections and resistance microbian in Intensive Care Unit of a University Hospital - an epidemiological study. Online Braz J Nurs. 2006;5(2). http://dx.doi.org/10.1590/ S0104-11692010000200014.

13. Portaria 2.048 de 05 de novembro de 2002. Aprova, na forma do anexo desta portaria, o regulamento técnico dos sistemas estaduais de urgência e emergência. Brasília; 05 nov. 2002. Disponível em: http://www.saude.mg.gov.br/index. php?option=com_gmg\&controller=document\&id=875.

14. Pires Dos Santos R, Konkewicz LR, Nagel F, Lisboa T, Jacoby T, Gastal SL, et al. The 2009 H1N1 Influenza A Pandemic and Hand Hygiene Practices in a Hospitalin the South of Brazil. Infect Control Hosp Epidemiol. 2010;31(12):1313-5. doi: 10.1086/657582.

15. Nascimento NB, Travassos CMR. O erro médico e a violação às normas e prescrições em saúde: uma discussão teórica na área de segurança do paciente. Physis. 2010;20(2):625-51. http://dx.doi.org/10.1590/S0103-73312010000200016.

16. Borges MG, Ribeiro LCM, Figueiredo LFS, Sirico SCA, Souza MA. Adesão à prática de higienização das mãos por profissionais de saúde de um Hospital Universitário. Rev Eletr Enf. 2010;12(2):266-71. Available from: http:// www.moreirajr.com.br/revistas.asp?fase $=$ r003\&id materia $=5183$.

17. Lee A, Chalfine A, Daikos GL, Garilli S, Jovanovic B, Lemmen $\mathrm{S}$, et al. Hand hygiene practices and adherence determinants in surgical wards across Europe and Israel: a multicenter observational study. Am J Infect Control.
2011;39(6):517-20. doi: 10.1016/j.ajic.2010.09.007.

18. Oliveira AC, Cardoso CS, Mascarenhas D. Contact precautions in intensive care units: facilitating and inhibiting factors for professionals' adherence. Rev Esc Enferm USP. 2010;44(1):161-5. http://dx.doi.org/10.1590/S008062342010000100023 .

19. Bathke J, Cunico PA, Maziero ECS, Cauduro FLF, Sarquis LMM, Cruz EDA. Infraestrutura e adesão à higienização das mãos: desafios à segurança do paciente. Rev Gaúcha Enferm. 2013;34(2):78-85. http://dx.doi.org/10.1590/ S1983-14472013000200010.

20. Eduardo KGTE, Ferreira ERM, Pinheiros AKB, Ximenes LB. Utilização do instrumento de melhoria de desempenho na avaliação do exame de Papanicolaou por enfermeiros. Cogitare Enferm. 2008;13(3):329-35. http://dx.doi. org/10.5380/ce.v13i3.12963.

21. Salmon S, Bùi DP, Pittet D, McLaws ML. Beginning the journey of hand hygiene compliance monitoring at a 2,100-bed tertiary hospital in Vietnam. Am J Infect Control. 2014;42(1):71-3. doi: 10.1016/j.ajic.2013.07.011.

22. Resolução-RDC N.42, de 25 de outubro de 2010. Dispõe sobre a obrigatoriedade de disponibilização de preparação alcoólica para friç̧ão antisséptica das mãos, pelos serviços de saúde do País, e dá outras providências. Brasília; 2010. Disponível em: http://www20.anvisa.gov. br/segurancadopaciente/index.php/legislacao/item/rdc-42de-25-de-outubro-de-2010. 